\title{
Erratum de : Irradiation du personnel en neuroradiologie interventionnelle pédiatrique : focus sur le cristallin de l'opérateur
}

\author{
C. Bolomey ${ }^{1}$, G. Fasel ${ }^{1}$, N. Ryckx ${ }^{2}$ and R. Le Coultre ${ }^{1 \star}$ \\ 1 Haute École de Santé Vaud (HESAV), Haute École Spécialisée de Suisse Occidentale (HES-SO), Lausanne, Suisse. \\ 2 Institut de Radiophysique (IRA), Centre Hospitalier Universitaire Vaudois (CHUV), Lausanne, Suisse.
}

Radioprotection 51(3), 163-170 (2016), DOI: 10.1051/radiopro/2016024

Received 4 November 2016

Des erreurs se sont glissées lors de la mise en page des Tableaux 3 et 4. Les valeurs sont corrigées dans cet erratum.

Table 3. Moyenne des équivalents de dose individuel $\mathrm{Hp}(0,07)$ au niveau des yeux en fonction de la localisation des TLD, ainsi que les facteurs d'exposition du cristallin obtenus en divisant le cumul de dose TLD par le cumul de PDS des trois groupes d'interventions tels que listés dans le Tableau 2. L'incertitude élargie $(k=2)$ liée au TLD s'élève à $11 \%$.

Average individual dose equivalents $\mathrm{Hp}(0.07)$ of the eyes as a function of the location of the TLDs, and the eye lens exposure factors obtained by dividing the cumulative TLD doses by the respective cumulative DAP of the three groups of procedures, as listed in Table 2 . The expanded uncertainty $(k=2)$ related to the TLD is $11 \%$.

\begin{tabular}{|c|c|c|c|}
\hline $\begin{array}{l}\text { Emplacement } \\
\text { des TLD }\end{array}$ & $\begin{array}{c}\text { Mesure } \\
\mathbf{n}^{\circ}\end{array}$ & $\begin{array}{c}\text { Moyenne Hp(0,07) } \\
{[\mu \mathrm{Sv}](\mathrm{CI} 95 \%)}\end{array}$ & $\begin{array}{l}\text { Facteur d'exposition } \\
\left.[\mu \mathbf{S v}] \mathbf{S v} /\left(\mathbf{G y} \mathbf{c m}^{2}\right)\right]\end{array}$ \\
\hline \multirow{3}{*}{ Gauche } & 1 & $141 \pm 7(136,146)$ & 1,2 \\
\hline & 2 & $134 \pm 8(129,139)$ & 1,2 \\
\hline & 3 & $218 \pm 10(212,224$ & 1,9 \\
\hline \multirow{3}{*}{ Centre } & 1 & $127 \pm 7(123,131)$ & 0,8 \\
\hline & 2 & $115 \pm 9(109,121)$ & 0,7 \\
\hline & 3 & $178 \pm 12(171,185$ & 1,1 \\
\hline \multirow{3}{*}{ Droite } & 1 & $51 \pm 16(41,61)$ & 0,3 \\
\hline & 2 & $28 \pm 9(22,34$ & 0,2 \\
\hline & 3 & $37 \pm 8(32,42$ & 0,2 \\
\hline \multicolumn{2}{|c|}{ Moy. gauche } & $457 \pm 27$ & 1,2 \\
\hline \multicolumn{2}{|c|}{ Moy. droite } & $268 \pm 30$ & 0,6 \\
\hline
\end{tabular}

Table 4. Atténuation des lunettes plombées en fonction de l'orientation de l'opérateur et du tube utilisé. Attenuation of the leaded glasses as a function of the operator's orientation and the tube used.

\begin{tabular}{|c|c|c|c|c|c|}
\hline \multirow{3}{*}{ Tube utilisé } & \multirow{3}{*}{ Lunettes $\mathbf{n}^{\circ}$} & \multicolumn{4}{|c|}{$\begin{array}{c}\text { Atténuation en fonction } \\
\text { de l'orientation de la tête } \\
\text { de l'opérateur }\end{array}$} \\
\hline & & \multicolumn{2}{|c|}{ Moyenne [\%] } & \multicolumn{2}{|c|}{ Écart type [\%] } \\
\hline & & $90^{\circ}$ & $45^{\circ}$ & $90^{\circ}$ & $45^{\circ}$ \\
\hline \multirow{4}{*}{ Frontal } & 1 & 8,5 & 11,8 & 0,8 & 0,4 \\
\hline & 2 & 4,0 & 10,7 & 2,9 & 0,6 \\
\hline & 3 & 39,8 & 23,7 & 0,2 & 1,9 \\
\hline & 4 & 53,6 & 28,4 & 0,7 & 1,2 \\
\hline \multirow{4}{*}{ Latéral } & 1 & 43,4 & 61,8 & 0,1 & 0,2 \\
\hline & 2 & 31,9 & 52,1 & 0,5 & 0,1 \\
\hline & 3 & 69,0 & 66,9 & 0,1 & 0,3 \\
\hline & 4 & 74,9 & 67,4 & 0,1 & 0,1 \\
\hline \multirow{4}{*}{ Biplan } & 1 & 23,5 & 34,6 & 0,2 & 0,6 \\
\hline & 2 & 13,9 & 23,4 & 0,9 & 0,3 \\
\hline & 3 & 52,2 & 44,8 & 0,5 & 0,5 \\
\hline & 4 & 63,1 & 43,6 & 0,1 & 0,6 \\
\hline
\end{tabular}

^ regis.lecoultre@hesav.ch 